\title{
AMPLIANDO LA PROTECCIÓN \\ A SEGUNDAS INDICACIONES EN EUROPA: \\ SWISS-TYPE CLAIM Y EUROPEAN-TYPE CLAIM
}

CARLOS AUguSTO CONDE-GUTIÉRREZ*

\section{INTRODUCCIÓN}

El artículo 27.1 de los Acuerdos de la Organización Mundial de Comercio (omc) sobre los Aspectos de los Derechos de Propiedad Intelectual relacionados con el Comercio (ADPICs) establece que se deben otorgar patentes y garantizar su goce sobre cualquier tipo de invención o tecnología sin importar la categoría de este y/o el lugar de invención ${ }^{1}$. Esta disposición busca que los Estados miembros de la OMC no discriminen determinadas tecnologías como lo venían haciendo algunos de ellos antes de la promulgación de los ADpics.

En efecto, a través de la historia, los Estados, incluyendo a aquellos de países en desarrollo, han negado patentes sobre particulares invenciones con el ánimo de facilitar la transferencia de tecnología. Dichas legislaciones, al no otorgar protección a inventores de determinadas industrias, permitían que sus nacionales pudiesen copiar y adaptarse a una determinada tecnología sin pagar el costo derivado de una licencia sobre una tecnología específica. Por ejemplo, Reino Unido, Suiza y Francia habían discriminado determinadas tecnologías para facilitar que productores locales pudiesen copiar la tecnología sin la autorización

\footnotetext{
* Docente investigador del Departamento de Propiedad Intelectual de la Universidad Externado de Colombia. Abogado de la Universidad Externado de Colombia, Bogotá, Colombia. Candidato a doctor en Derecho (Ph.D.) y MPhil por la Universidad de Sheffield, Reino Unido. Máster en Biotecnología, Ética y Derecho. Contacto: carlos.conde@ uexternado.edu.co

Fecha de recepción: 4 de abril de 2015. Fecha de aceptación: 2 de mayo de 2015. Para citar el artículo: Conde-Gutiérrez, C. A. Ampliando la protección a segundas indicaciones en Europa: 'Swiss-type Claim' y 'European-type Claim'. Revista La Propiedad Inmaterial n. ${ }^{\circ}$ 19, Universidad Externado de Colombia, enero-junio, 2015, pp. 77-88. DOI: 10.18601/16571959.n19.04

1. Acuerdo sobre Aspectos de los Derechos de Propiedad Intelectual Relacionados con el Comercio (ADpics), Anexo 1c del Acuerdo de Marrakech por el que se establece la OMC.
} 
del titular ${ }^{2}$. Este tipo de prácticas se vieron en especial en la industria química, y posteriormente en la industria farmacéutica ${ }^{3}$. Sin embargo, con la estandarización de los requisitos de patentes y la vinculación del comercio internacional con la propiedad intelectual, no solo se eliminó la facultad de los Estados de discriminar el otorgamiento de patentes a determinadas tecnologías o invenciones (art. 27.1 de los ADPIC), como las químicas o farmacéuticas, sino que se ha ido ampliando el ámbito de protección de este tipo de invenciones ${ }^{4}$.

En efecto, los países desarrollados, en especial los de Europa, han ampliado el ámbito de protección de patentes a través de figuras como las de patentes sobre primeras y segundas indicaciones médicas. Las patentes sobre primeras indicaciones médicas surgen ante la necesidad de otorgar patentes respecto de reivindicaciones de uso a una composición o sustancia química que ya es conocida debido a que la industria química es precursora de la industria farmacéutica; así, muchas de las composiciones y sustancias utilizadas en la industria química fueron y han sido empleadas para el tratamiento de enfermedades 5 . Por lo tanto, en el caso de este tipo de sustancias o composiciones, lo que se protege por medio de una reivindicación en una patente es el uso que a dicha sustancia o composición química se le da en el tratamiento de una determinada enfermedad.

Adicionalmente, la segunda indicación o segundo uso se refiere a la misma composición o sustancia empleada con fines farmacéuticos, pero con un uso diferente a la primera indicación, y se conoce como Swiss-type Claim. Sin embargo, la Oficina de Patentes Europea (EPO, por sus siglas en inglés) ha diseñado un nuevo tipo de reivindicación que incluye en principio las segundas indicaciones en la forma de Swiss-type Claim, al igual que otros usos sobre la misma sustancia o composición (i.e., terceras o cuartas indicaciones), la cual se ha denominado European-type Claim.

Pese a esta amplitud que se ha generado en Europa, para proteger segundas o terceras o más indicaciones, en el caso de la Comunidad Andina el artículo 21 de la Decisión 486 de 2000 (Régimen Común sobre Propiedad Industrial) proscribe las patentes sobre segundos usos o indicaciones ${ }^{6}$. El artículo en cita establece: "Los productos o procedimientos ya patentados, comprendidos en el estado de la técnica, de conformidad con el artículo 16 de la presente Decisión, no serán

2. C. May y S. Sell, Intellectual Property Rights: a Critical History (Lynne Rienner, 2006).

3. D. Burk y M. Lemley, 'Policy Levers in Patent Law' [2003] 89 Virginia Law Review 1575

4. Para una dicusión crítica sobre el rol del comercio internacional en patentes y transferencia de tecnología en países en vía de desarrollo, ver A. JAMEs, Fairness in Practice (2012, Oxford University Press).

5. D. Schneider, 'Patenting of Pharmaceuticals - Still a Challenge?' [2008] 39 IIC 511-525

6. Decisión 486 de 2000, Régimen Común sobre Propiedad Industrial de la Comunidad Andina, Lima, 14 de septiembre de 2000. 
objeto de nueva patente, por el simple hecho de atribuirse un uso distinto al originalmente comprendido por la patente inicial" (resaltado nuestro).

La prohibición del artículo 21 ha sido igualmente ratificada por el Tribunal Andino de Justicia. En efecto, la Secretaría de la Comunidad Andina llevó ante el Tribunal Andino a Perú en $1999^{[7]}$ y a Venezuela ${ }^{8}$ (en ese entonces miembro de la Comunidad Andina) y a Ecuador ${ }^{9}$ en 2001, por permitir patentes sobre segundas indicaciones, y en los tres casos el Tribunal encontró que se había violado la prohibición andina. Igualmente, en Colombia, tanto la Superintendencia de Industria y Comercio (sIC) como el Consejo de Estado negaron una patente sobre una segunda indicación al considerar que estaba en clara contradicción con el requisito de novedad consagrado en los artículos 16 y 21 de la Decisión 486.

El presente trabajo busca analizar los aspectos técnicos y legales de las reivindicaciones en patentes sobre segundas indicaciones o usos en invenciones farmacéuticas. El análisis se centra principalmente en el Convenio sobre Patentes Europeo $^{10}$, las decisiones de la EPO y, en particular, las reivindicaciones conocidas como Swiss-type Claim y European-type Claim. Igualmente, se hace un análisis comparativo con la legislación y la jurisprudencia andinas y colombianas sobre este tema.

El artículo se divide en tres partes. La primera parte hace un breve recuento de las razones por las cuales la EPo adoptó el Swiss-type Claim. En esta parte se analizan los trabajos preparatorios del EPC, en donde se puede establecer que la excepción a la patentabilidad sobre métodos de tratamiento terapéutico y quirúrgico, y sobre métodos de diagnóstico sobre animales y humanos, sirvió como punto de partida, y a manera de compensación para la industria farmacéutica, para otorgar primero patentes acerca de reivindicaciones sobre primeras indicaciones en composiciones y sustancias ya conocidas. En la segunda parte se analiza cuál es alcance de la interpretación EPO a esta excepción, en la cual se consideran válidas no solo las reivindicaciones sobre primeras indicaciones o usos, sino también las segundas indicaciones o Swiss-type Claim. Igualmente, se estudia cómo la EPo ha ampliado el alcance del Swiss-type Claim con el ánimo de incluir no solo segundas indicaciones, sino también subsecuentes indicaciones sobre sustancias o composiciones ya conocidas en el estado de la técnica. En la tercera parte se analizan la prohibición del artículo 21 de la Decisión 486, los diferentes fallos del Tribunal Andino de Justicia, la regulación de la sic y un fallo del Consejo

7. Proceso n. ${ }^{\circ}$ 07-AI-1999.

8. Proceso n. ${ }^{\circ}$ 01-AI-2001.

9. Proceso n. ${ }^{\circ}$ 34-AI-2001.

10. Convenio de Múnich sobre Concesión de Patentes Europea, de 5 de octubre de 1973 (versión consolidada tras la entrada en vigor del Acta de revisión de 2000). 
de Estado colombiano, los cuales se distancian del Convenio Europeo y de las decisiones de la EPO.

\section{Antecedentes del SWISS-Type Claim}

La industria farmacéutica europea siempre ha resaltado la importancia de incentivar la investigación y el desarrollo $(\mathrm{I}+\mathrm{D})$ a través de mecanismos de protección exclusiva como las patentes o los certificados complementarios de protección ${ }^{11}$. Este último busca compensar al titular de una patente el periodo de tramitación en la obtención de un registro sanitario para comercialización, con un periodo complementario de protección sobre la invención objeto de patente ${ }^{12}$.

Antes de la promulgación del Convenio Europeo sobre Patentes en 1973, en sus trabajos preparatorios se puede observar cómo los países integrantes de las comisiones negociadoras llegaron al acuerdo de considerar que los métodos de tratamiento terapéutico y quirúrgico y los métodos de diagnóstico sobre animales y humanos, por razones de salud pública no podían ser considerados objeto de aplicación industrial, ya que diferentes países europeos, como Alemania, buscaban proteger la actividad de los profesionales de la salud y de los veterinarios ${ }^{13}$. Esta ficción legal, por la cual se consideraba que este tipo de invenciones carecían del requisito de aplicación industrial, se encontraba establecida en el artículo 52.4 del Convenio de 1973.

Sin embargo, el lobby de las compañías farmacéuticas en Europa logró conseguir, a manera de compensación, que la excepción establecida en el artículo 52.4 del Convenio de 1973 no se aplicara "a los productos, en particular las sustancias o composiciones, para la aplicación de uno de estos métodos”. En otras palabras, los métodos descritos en el artículo 52.4 del Convenio de 1973 no son patentables, pero las sustancias o composiciones que se emplean o utilizan en cualquier método terapéutico son patentables.

La norma del artículo 52.4 del Convenio de 1973 fue enmendada más adelante por la versión revisada del Convenio Europeo en el año 2000, en la que simplemente se encasilló entre las excepciones a la patentabilidad (art. 53(c)) y no entre las invenciones que carecían de aplicabilidad industrial.

En la versión de 1973 y la revisada de 2000, el artículo 54.4, que regula la novedad en las patentes, va más allá al manifestar la posibilidad de obtener una

11. efpia, 2013 Annual Review and Forward Look (2012, European Federation of Pharmaceutical Industries and Associations).

12. Ver Reglamento (CE) n.o $469 / 2009$ del Parlamento Europeo y del Consejo relativo al Certificado Complementario de Protección para los Medicamentos.

13. Directorate Patent Law, “Article 52", en Travaux Préparatori (EPC 1973) (EPO, 1973) disponible en: http://webserv.epo.org/projects/babylon/tpepc73.nsf/0/719AC39AA49A75 63C12574270049EB9E/\$File/Art52eTPEPC1973.pdf Consultado el 22 de enero 2015. 
patente sobre sustancia o composición ya conocidas para la utilización en los métodos descritos en el artículo 53 (c) de la versión revisada de 2000. Esto indica que se permite la patentabilidad sobre el uso en el tratamiento de una enfermedad, a pesar de que las sustancias y composiciones empleadas carezcan, en principio, de novedad, siempre y cuando la utilización no se encuentre en el estado de la técnica.

Por lo tanto, en principio, el artículo 54.4 permite la patentabilidad de primeras indicaciones sobre sustancias o composiciones utilizadas en los métodos descritos en el artículo 53 (c). Esto es lo que se conoce como primera indicación médica, la cual señala el uso que se le debe dar a una sustancia o composición (i.e., medicamento) para el tratamiento de una enfermedad.

No obstante la posibilidad de obtener patentes sobre primeras indicaciones médicas en sustancias y composiciones ya conocidas (art. 54 (4)), la EPO extendió el alcance del artículo 53 (c) no solo a primeras indicaciones sino también a segundas indicaciones o Swiss-Type Claim (art. 54 (5)). La siguiente sección explica con más detalle la interpretación por parte de la EPO de los artículos 53 (c) y 54 (4) (5) sobre segundas indicaciones médicas, y la manera como recientes interpretaciones han extendido la patentabilidad tanto a segundas indicaciones como a subsecuentes indicaciones.

\section{Del SWISS-TYPE CLAIM al EUROPEAN-TYPE CLAIM}

Para poder entender el alcance de las reivindicaciones tipo suizo o Swiss-type Claim, es importante observar cómo estas patentes son redactadas en Europa. Una reivindicación tipo suizo generalmente se redacta, en una solicitud de patentes, bajo la forma: "el uso de x en la fabricación de un medicamento para el tratamiento de $\mathrm{Y}^{\text {"14. }}$. Como se puede observar, la reivindicación se enfoca en el uso, no en la sustancia ya existente para el tratamiento de una determinada enfermedad. Por lo tanto el problema de este tipo de revindicaciones es el de cómo demostrar que el segundo uso, y no el producto en sí, no está en el estado de la técnica.

Por lo tanto, aunque las patentes sobre segundas indicaciones tienen su origen como una excepción dentro de la excepción de los métodos de tratamiento terapéutico y quirúrgico, y de los métodos de diagnóstico sobre animales y humanos, este tipo de reivindicaciones están igualmente relacionadas con el requisito de novedad. En efecto, el artículo 54 (4) del Convenio de 1973 establece que no se puede excluir de patentabilidad el uso de una composición o sustancia, ya conocida en el estado de la técnica, sobre los métodos de tratamiento terapéutico y quirúrgico, y sobre los métodos de diagnóstico sobre animales y humanos. El análisis de casos de la EPO nos permitirá entender mejor este punto.

14. J. Pila, 'Methods of Medical Treatment within Australian and United Kingdom Patents Law' [2001] 24 UNSW Law Journal 420. 
Primeras indicaciones y SWISS-TyPE CLAIM en el Convenio DE 1973

La EPO en EISA/Second Medical Indication ${ }^{15}$ permitió, bajo una interpretación extensiva del Convenio de 1973 (arts. 52 (4) y 54 (5)), la patentabilidad de segundas indicaciones, incluyendo el uso de una sustancia o composición conocida, en un nuevo método de tratamiento terapéutico. Como se mencionó anteriormente, la versión original del Convenio de 1973 establecía en su artículo 52 (4) una ficción legal de la falta de aplicabilidad industrial sobre métodos de tratamiento terapéutico y quirúrgico, y sobre los métodos de diagnóstico sobre animales y humanos. Sin embargo, la EPO en EISA/Second Medical Indication aclara que dicha prohibición no recae sobre las sustancias o composiciones utilizadas en el tratamiento de los métodos descritos en el artículo 52 (4) del Convenio de 1973, los cuales sí son susceptibles de aplicación industrial ${ }^{16}$.

Adicionalmente, la EPO aclara que respecto al requisito de novedad, el artículo 54 (5) claramente establece que no se debe excluir de patentabilidad ninguna sustancia o composición para su uso en cualquiera de los métodos descritos en el artículo 52 (4), inclusive si dichas sustancia o composición ya se encuentra en el estado actual de la técnica ${ }^{17}$. Según la EPO, esto es lo que se conoce como primera indicación médica, donde el inventor puede obtener protección sobre un producto de propósito limitado ${ }^{18}$. En otras palabras, EISA/Second Medical Indication aclara que un inventor puede obtener una patente sobre el primer uso de una sustancia o composición que ya existe en el estado de la técnica para el tratamiento de una enfermedad; en ese sentido, la invención estaría limitada a ese primer uso (o propósito limitado) en el tratamiento de una enfermedad.

Respecto a segundas indicaciones, la EPo cita a la Sala Civil de la Corte Federal de Justicia de Alemania en el caso Hydropyridin, el cual permitió las patentes sobre segundas indicaciones con una redacción específica ${ }^{19}$. En este caso la Corte Federal establece que, de acuerdo a la ley alemana, una reivindicación puede ser redactada de manera tal que el uso de una sustancia química para el tratamiento de una enfermedad se extienda más allá del tratamiento de la enfermedad, por lo que se puede incluir, por lo menos, la forma en que dicha sustancia es empacada con instrucciones sobre el uso para el tratamiento de la enfermedad. Este tipo de

15. G 05/83 EISA/Second Medical Indication oJepo 1985, 64 .

16. Ibíd., párr. 15 .

17. Ibíd.

18. Ibíd.

19. Caso x zв 4/ 1983 Hydropyridine, Sala Civil de la Corte Federal de Justicia de Alemania. 
reivindicación, por lo tanto, puede usarse como base para solicitar protección a segundas (o inclusive otras) indicaciones.

Igualmente, la EPO refuerza el argumento de la posibilidad de obtener protección a segundas indicaciones con la práctica de la Oficina de Patentes Suiza, la cual protege segundas indicaciones por medio de reivindicaciones sobre el uso de una sustancia o composición para la fabricación de un medicamento para una nueva aplicación terapéutica específica ${ }^{20}$.

Finalmente, la EPO aclara que el requisito de novedad se cumple en segundas indicaciones no por el producto en sí, sino por el nuevo uso farmacéutico que se le da a dicho producto ${ }^{21}$. Esto quiere decir que el artículo 54 (5) del Convenio de 1973 es no solo la vía para validar el requisito de novedad en primeras indicaciones, sino que también se extiende a segundas indicaciones.

En este sentido, un Swiss-type Claim que incluya un método de tratamiento terapéutico podría redactarse de la siguiente manera: "el uso de una sustancia o composición en la fabricación de un medicamento para una aplicación terapéutica específica, nueva e inventiva" ${ }^{22}$. Sin embargo, la versión modificada del Convenio del año 2000 ha llevado a la EPO a encontrar una interpretación más amplia respecto a las segundas indicaciones.

\section{THE EUROPEAN-TYPE CLAIM}

La versión del Convenio del año 2000 busca clarificar el alcance del artículo 54 (5) de la versión de 1973 respecto al alcance de las primeras y segundas (o terceras o sucesivas) indicaciones. En efecto, el nuevo artículo 54 (4) del Convenio del año 2000 mantiene la redacción del artículo 54 (5) sobre el cual se permite las primeras indicaciones respecto de la nueva utilización de una sustancia o composición que ya se encuentra en el estado de la técnica, esto es, primeras indicaciones. Respecto a las segundas indicaciones, el artículo 54 (5) de la versión revisada de 2000 considera patentable una nueva "utilización específica" sobre una sustancia o composición que se encuentra en el estado de la técnica y sobre la cual ya existe una primera indicación.

En Abbott Respiratory/Dossage Regime, la Epo señala que la nueva redacción del artículo 54 (4) permite asegurar la patentabilidad de manera más clara sobre las sustancias y composiciones conocidas sobre usos en métodos relacionados en el artículo 53 (c) (art. 54 (4) del Convenio de 1973), o primera indicación, y la patentabilidad de las sustancia y composiciones conocidas sobre usos "específicos" (producto limitado) en los métodos establecidos en el artículo 53 (c); esto es,

20. N. 17, párr. 19 .

21. Ibíd., párr. 20-22.

22. Ibíd., párr. 23. 
protección a las segundas indicaciones (art. 54 (5)); en ambas hipótesis, dichos usos deben ser considerados nuevos ${ }^{23}$.

En este sentido, las oficinas de patentes locales, como la de Reino Unido, han considerado que con base en la nueva redacción e interpretación del artículo 54 (5), las reivindicaciones sobre segundas indicaciones deben ser redactadas así: "sustancia X para uso en el tratamiento de la enfermedad Y"24. De esta manera, la EPo señaló que el Swiss-type Claim debe considerarse incluido dentro de la nueva redacción del artículo 54 (4) (5), puesto que esta recoge usos sobre primeras y segundas indicaciones. Esto es lo que se ha denominado recientemente el European-type Claim.

Sin embargo, en Board of Regents/Cancer Treatment, la EPO ha agregado más complicaciones a la interpretación de este tipo de reivindicaciones, al considerar que tanto el Swiss-type Claim como el European-type Claim pueden coexistir en una misma aplicación ${ }^{25}$. En efecto, la EPO ha establecido que reivindicaciones cuya redacción comprenda la fórmula "el uso de X en la fabricación de un medicamento para el tratamiento de Y" (Swiss-type Claim) están relacionadas sobre un proceso de propósito limitado ${ }^{26}$. En otras palabras, si una reivindicación está redacta en la forma de un Swiss-type Claim, se entiende que esta recae sobre el proceso (i.e., la fabricación de un medicamento). Por esta razón, la EPO encuentra que una reivindicación redactada como "sustancia $X$ para uso en el tratamiento de la enfermedad Y” (European-type Claim) es diferente a la Swiss-type Claim, debido a que esta última recae sobre la fabricación del medicamento, mientras que la Europea-type Claim versa sobre una reivindicación de producto (o uso) de propósito limitado, por lo que ambas pueden coexistir en una misma patente ${ }^{27}$.

La evolución legislativa y casuística europea ilustra el gran interés de la EPO en proteger primeras y segundas (o terceras o sucesivas) indicaciones en sustancias o composiciones para el tratamiento de enfermedades. Esto demuestra claramente que es vital para la EPO proteger de manera generosa las invenciones provenientes de la industria farmacéutica europea. Dicha postura es aún más evidente en Board of Regents/Cancer Treatment, porque no solo se protege el nuevo uso específico (producto de propósito limitado o European-type Claim) de una sustancia o composición ya existente, sino también el proceso de fabricación de la misma sustancia o composición (un proceso de propósito limitado o Swiss-type Claim). Semejante interpretación claramente busca beneficiar a la industria farmacéutica.

23. G 02/08 Abbott Respiratory/Dossage Regime ojepo 2010, 456.

24. Ipo, Examination Guidelines for Patent Applications relating to Medical Inventions in the IPO (2013, IPO).

25. T 1780/12 Board of Regents/Cancer Treatment.

26. Ibíd., párr. 16.

27. Ibíd., párr. 16 y 17. 


\title{
Segundas indicaciones en la Comunidad Andina
}

Aunque la Epo ha interpretado generosamente el Convenio con el fin de garantizar patentes sobre segundas (o terceras o sucesivas) indicaciones, la Comunidad Andina parece ser mucho más restrictiva respecto de este tipo de reivindicaciones. En efecto, el artículo 21 de la Decisión 486 de 2000 establece que no se puede considerar un procedimiento o producto nuevo "por el simple hecho de atribuirse un uso distinto al originalmente comprendido por la patente inicial". Claramente el citado artículo 21 elimina la posibilidad de que un nuevo uso específico sobre una composición o sustancia ya existente en el estado de la técnica pueda ser objeto de patentabilidad.

El caso de Viagra de Pfizer en la Comunidad Andina ilustra el alcance del artículo 21 de la Decisión 486 en la CaN. Pfizer intentó obtener una patente sobre una segunda indicación respecto a la composición Pyrazolopyrimidines. Esta segunda indicación se comercializa bajo el nombre de Viagra en varios países de la Comunidad Andina (Colombia, Ecuador, Perú y Venezuela). La primera indicación para este producto era para tratar infecciones cardiovasculares y el segundo para el tratamiento de la disfunción eréctil.

Aunque el artículo 21 de la Decisión 486 indica que no debe haber patentes sobre segundas indicaciones o usos, Ecuador ${ }^{28}$, Venezuela ${ }^{29}$ y Perú buscaron otorgar patentes sobre la composición Pyrazolopyrimidines para tratar la erección disfuncional. Como resultado de ello, la Secretaría de la CAN tomó los tres países ante el Tribunal de Justicia Andino.

Para el Tribunal es claro "e"?que en la Comunidad Andina se excluye la segunda indicación, cuando menciona que la norma andina "subordina la no concesión de una nueva patente, al hecho de que los productos o los procedimientos ya patentados se encuentran innegablemente comprendidos en el estado de la técnica; estado éste que, por otra parte, se constituye de manera automática con el otorgamiento de la patente original" 30 .

$\mathrm{Al}$ establecer un criterio de novedad tan estricto, para el Tribunal es inequívoca la regulación andina frente a la prohibición de otorgar patentes de proceso o producto sobre segundas indicaciones. Por lo tanto, en la Comunidad Andina no cabría ni el Swiss-type Claim ni el European-type Claim en ninguna de las formas en que la EPO ha interpretado los dos tipos de reivindicaciones.

Lo anterior significa: primero, que no puede existir una patente sobre un nuevo procedimiento de una sustancia o composición que se encuentre en el estado de la técnica (fabricación de medicamento o proceso de propósito limitado, Swiss type-Claim), y segundo, que no hay lugar dentro de la Comunidad Andi-

\author{
28. N. 9. \\ 29. N. 7. \\ 30. N. 9, p. 31 .
}


na a otorgar una patente sobre un nuevo uso específico (producto de propósito limitado o European-type Claim) de una sustancia o composición ya existente.

Finalmente, en cuanto a Colombia, su oficina de patentes (sIC) siguió los lineamientos del Tribunal Andino de Justicia, y no concedió una patente sobre segunda indicación de Pfizer $^{31}$; tal decisión fue confirmada por el Consejo de Estado de Colombia en $2008^{[32]}$.

\section{Conclusiones}

La evolución conceptual de las segundas indicaciones o usos de sustancias o composiciones ya conocidas en el estado del arte en Europa, demuestra un gran interés por parte de la EPO en otorgar un alto grado de protección a productos y procesos farmacéuticos que no necesariamente sean nuevos, pero tengan un uso diferente. Este interés es aún más evidente en Board of Regents/Cancer Treatment, en donde la EPO no solo ha otorgado protección a segundas (o terceras o sucesivas) indicaciones sobre usos específicos (producto de propósito limitado o European-type Claim), sino también sobre nuevos procesos (fabricación de un medicamento o Swiss-type Claim) en una misma composición o sustancia ya conocida en el estado del arte.

Entre tanto, la regulación de la Decisión 486, junto con las decisiones del Tribunal Andino de Justicia, la sic y el Consejo de Estado colombiano, permiten concluir que tanto en la Comunidad Andina como en Colombia las segundas indicaciones en procesos y productos no pueden ser consideradas patentables. Esto claramente beneficia no solo a las compañías productoras de medicamentos genéricos, que pueden acceder a las sustancias y composiciones que se encuentran en el estado del arte, sin importar si existen nuevos usos, sino también a pacientes en los países andinos, quienes pueden acceder rápidamente no solo a las primeras indicaciones sino también a los demás usos y procesos que recaigan sobre las composiciones y sustancias conocidas en el estado del arte.

\section{BIBLIOGRAFÍA}

\section{LEGISLACIÓN}

Acuerdo sobre Aspectos de los Derechos de Propiedad Intelectual Relacionados con el Comercio (ADPICs), Anexo 1c del Acuerdo de Marrakech por el que se establece la OMC. 
Decisión 486 de 2000, Régimen Común sobre Propiedad Industrial de la Comunidad Andina, Lima, 14 de septiembre de 2000.

Convenio de Múnich sobre Concesión de Patentes Europea, de 5 de octubre de 1973.

Convenio de Múnich sobre Concesión de Patentes Europea, de 5 de octubre de 1973 (versión consolidada tras la entrada en vigor del Acta de revisión de 2000). Reglamento (CE) n. ${ }^{\circ}$ 469/2009 del Parlamento Europeo y del Consejo Relativo al Certificado Complementario de Protección para los Medicamentos.

Jurisprudencia y casos Oficinas de Patentes

\section{Comunidad Andina y Colombia}

Proceso n. ${ }^{\circ} 07-\mathrm{AI}-1999$.

Proceso n. ${ }^{\circ} 01-\mathrm{AI}-2001$.

Proceso n. ${ }^{\circ}$ 34-AI-2001.

Superintendencia de Industria y Comercio, Resolución 112 del 18 de enero de 2000.

Pfizer Research and Development Company vs. Superintendencia de Industria y Comercio (2008, Consejo de Estado, Ref.: 2000-066008).

\section{Europa}

Caso x zв 4/ 1983 Hydropyridine, Sala Civil de la Corte Federal de Justicia de Alemania.

G 05/83 eIsA/Second Medical Indication oJepo 1985, 64.

G 02/08 Abbott Respiratory/Dossage Regime ojepo 2010, 456.

$\mathrm{T}$ 1780/12 Board of Regents/Cancer Treatment.

\section{DOCTRINA}

Burk, D. \& M. Lemley, 'Policy Levers in Patent Law' [2003] 89 Virginia Law Review 1575.

Directorate Patent Law, 'Article 52', en Travaux Préparatori (EPC 1973) (epo, 1973), disponible en: http://webserv.epo.org/projects/babylon/tpepc73.nsf /0/719AC39AA49A7563C12574270049EB9E/\$File/Art52eTPEPC1973. pdf Consultado el 22 de enero 2015.

EFPIA, 2013 Annual Review and Forward Look (2012, European Federation of Pharmaceutical Industries and Associations).

IPO, Examination Guidelines for Patent Applications relating to Medical Inventions in the IPO (IPO, 2013). 
James, A., Fairness in Practice (Oxford University Press, 2012),

May y Sell, S., Intellectual Property Rights: A Critical History (Lynne Rienner, 2006).

Schneider, D., 'Patenting of Pharmaceuticals - Still a Challenge?' [2008] 39 IIC 511-525.

Pila, J., 'Methods of Medical Treatment within Australian and United Kingdom Patents Law' [2001] 24 UNSW Law Journal 420. 